\section{DOI: 10.1007/s00350-010-2714-1}

\section{Ärztlicher Behandlungsfehler und schicksalhafter Verlauf.}

Von Ralph B. Seifert. Verlag Nomos, Baden-Baden 2008, 200 S., kart., $€ 46,00$

Die mit dem Nachweis des Kausalzusammenhanges zwischen ärztlichem Behandlungsfehler und Rechtsgutsverletzung des Patienten verbundenen Schwierigkeiten sind seit langem bekannt. In jüngster Zeit mehrt sich jedoch die Kritik am Lösungsansatz der Rechtsprechung, die Beweislast bei groben Behandlungsfehlern umzukehren. Ein alternatives Haftungskonzept, welches Schadensersatz bereits be nur möglicher Verursachung zulässt, die sog. Wahrscheinlichkeitshaftung, beschäftigte den 66. Deutschen Juristentag. Seifert unterstützt in seiner von Eike Schmidt betreuten Bremer Dissertation diesen Vorschlag, auf den Kausalitätsnachweis zur vollen Überzeugung des Gerichts zu verzichten und im Gegenzug den Ersatzanspruch des Patienten entsprechend der Verursachungswahrscheinlichkeit zu kürzen.

Zu Beginn geht der Verfasser grundlegend auf den Kausalzusammenhang als Voraussetzung ärztlicher Haftung ein. Nennenswer ist hier die Auseinandersetzung mit wissenschaftstheoretischen Erkenntnissen zur Kausalität (S. 39). An deren Ende steht die Feststellung, dass die Verursachung praktisch nie mit absoluter Gewissheit ermittelt werden könne, sondern allenfalls eine Wahrscheinlichkeitsaussage möglich sei. Es folgt eine eingehende und anschauliche Darstellung des zuvor aufgeworfenen Begriffs der Wahrscheinlichkeit. Im klassischen Sinne bewiesen sei die Kausalität erst, wenn die Wahrscheinlichkeit der Verursachung ausreichend hoch ist, um da Regelbeweismaß zu erfüllen. Zu diesem Ergebnis gelangt Seifert in dem nachfolgenden Kapitel zum Beweisrecht, das ein stark wahrscheinlichkeitsorientiertes Beweismaßverständnis erkennen lässt. Eine Reduzierung des Beweismaßes auf die überwiegende Wahrscheinlichkeit wird hingegen nicht befürwortet.

Im Mittelpunkt der zweiten Hälfte der Arbeit stehen die Lösungsmöglichkeiten für das aus den Unwägbarkeiten einer medizinischen Behandlung folgende „Kausalitätsdilemma.“ Die von der Rechtsprechung praktizierte Beweislastumkehr ist nach Meinung des Verfassers weder ausreichend begründet noch sachgemäß (S. 135). Die Schwäche einer jeden beweisrechtlichen Lösung liege darin, dass sie dem „Alles-oder-Nichts-Prinzip“ verhaftet bleibe und den verfügbaren Wahrscheinlichkeitserkenntnissen hinsichtlich der Kausalität keine ausreichende Bedeutung einräume. Vorzugswürdig sei es daher, eine anteilige Haftung zuzulassen, welche das Wissen über die Verursachungswahrscheinlichkeit unmittelbar anwende. Der Verfasser erkennt zwar an, dass die Rolle des medizinischen Sachverständigen dadurch noch an Bedeutung zunimmt, sieht darin jedoch keinen Nachteil.

Als dogmatischer Ansatzpunkt für eine Proportionalhaftung werden die Figur des Ersatzes von Heilungschancen sowie die aus dem Strafrecht bekannte Risikoerhöhungslehre nachvollziehbar verworfen. Hingegen verwundert die Ablehnung einer Analogie zu $₫ 830$ Abs. 1 S. 2 BGB mit der im Grunde einzigen Begründung, diese hätte anderenfalls einen zu weiten Anwendungsbereich und würde in allen Fällen zweifelhafter Verursachung zu einer anteiligen Haftung führen (S. 162). Denn auch die im Ergebnis befürwortete Lösung über die Fortsetzung des Rechtsgedankens aus $₫ 254$ Abs. 1 BGB knüpft an eine nicht spezifisch arzthaftungsrechtliche Norm an. Eine Beschränkung des Anwendungsbereiches ist hier ebenso erforderlich und wird vom Verfasser auch formuliert (S. 163). Die Möglichkei selbigen Vorgehens wird hinsichtlich der Analogie allerdings nicht erwogen. Gleichwohl schlägt Seifert hier den Weg ein, über welchen sich eine Abkehr vom Alles-oder-Nichts-Prinzip im Rahmen des geltenden Rechts noch am ehesten begründen ließe. Dass es sich dennoch um eine recht gestreckte Konstruktion handelt, zeigt bereits, dass dazu die „Fortsetzung eines Rechtsgedankens“ erforderlich wird.

Abschließend werden einige Fragen der Anwendung einer Wahrscheinlichkeitshaftung diskutiert. Interessant ist hier vor allem der Vorschlag, über $\$ 92$ Abs. 2 Nr. 2 ZPO das Risiko des Klägers abzufangen, die Prozesskosten teilweise tragen zu müssen, falls das Ge- richt die im Klageantrag bezifferte Verursachungswahrscheinlichkeit und folglich auch die Klageforderung für zu hoch hält (S. 175).

Insgesamt zeichnet sich die Arbeit von Seifert dadurch aus, dass sie die Problematik fundiert aufarbeitet und dabei stets die Besonderheiten der Arzthaftung im Blick behält. Zur Begründung einer derart gewichtigen Abkehr von haftungsrechtlichen Grundprinzipien fällt die Auseinandersetzung mit den bestehenden Kritikpunkten jedoch relativ knapp aus. Beispielsweise bleibt die Vereinbarkeit einer Schadensteilung mit dem schadensersatzrechtlichen Ausgleichsprinzip unerwähnt. $\mathrm{Ob}$ die Rechtsprechung gleichwohl dem Ruf nach einer Proportionalhaftung in solchen Konstellationen nachkommt und sie damit für die Praxis bedeutsam wird, bleibt abzuwarten.

\section{Martis/Winkhart: Arzthaftungsrecht - Fallgruppenkommentar.}

Von Rüdiger Martis und Martina Winkhart-Martis. Verlag Dr. Otto Schmidt, 3. Aufl. Köln 2010, 1231 S., geb., €89,80

Bereits rund drei Jahre nach der zweiten Auflage lohnte sich in Anbetracht der Fortentwicklung des zu Recht als „Richterrecht“ umschriebenen Arzthaftpflichtrechts durch die Rechtsprechung die Erarbeitung einer Neuauflage. Was Rüdiger Martis und Martina Winkhart-Martis im Zuge der Überarbeitung ihres Werks indes erneut geleistet haben, geht weit über das hinaus, was man sich üblicherweise von Neuauflagen zu erhoffen wagt. Die Autoren haben nicht nur die in der Zwischenzeit ergangene Rechtsprechung - nach eigenen Angaben über 600 Entscheidungen - akribisch ausgewertet und eingearbeitet, selbiges für das neu veröffentlichte Schrifttum unternommen und Anregungen aus dem Leser- und Rezensentenkreis aufgegriffen. Vielmehr wurde das gesamte Werk grundlegend überarbeitet und zahlreiche Abschnitte maßgeblich erweitert. Im Ergebnis ist der Umfang abermals von 918 auf 1197 Seiten gestiegen. Seit der Erstauflage hat sich die Seitenzahl damit - ungeachtet der bereits mit der Vorauflage erfolgten Umstellung auf ein größeres Format mit gebrauchsfreundlichem festem Einband - nahezu exakt verdoppelt. Zur Beibehaltung der Übersichtlichkeit und Gewährleistung einer zügigen Orientierung wurden die umfangreicheren Kapitel mit Zwischenüberschriften und das gesamte Werk mit Randziffern versehen.

Diese bereits rein äußerlich beeindruckende Leistung bewundert man um so mehr, wenn man das Werk im Arbeitsalltag zu Rate zieht. Der bewährten alphabetischen Aufgliederung des Arzthaftungsrechts in durch ein alphabetisches und ein systematisches Inhaltsverzeichnis leicht auffindbare Stichworte - von „AGB“ bis „,voll beherrschbare Risiken“ - folgend behandeln die Autoren sämtliche in der Rechtspraxis regelmäßig auftretenden materiell- und prozessrechtlichen Problembereiche des in tatsächlicher und rechtlicher Hinsicht anspruchsvollen Rechtsgebiets in anschaulicher, verständlicher und informativer Weise. Ob Fragen der ärztlichen Aufklärung, der Behandlungsfehlerhaftung, des Beweis- oder des Verjährungsrechts - stets findet der Leser zuverlässige und umfassende Ausführungen vor. Aufgrund der das gesamte Werk beherrschenden hohen Qualität können einzelne Passagen nur beispielhaft hervorgehoben werden. Mit Blick auf die Erweiterungen der Neuauflage sei an dieser Stelle jedoch insbesondere auf die bemerkenswerten Aktualisierungen zur Anknüpfung der Haftung an eine unterlassene Befunderhebung unter Einschluss der Frage der Abgrenzung zum Diagnoseirrtum sowie auf die imposante, nochmals ausgeweitete Darstellung des Berufungsrechts aufmerksam gemacht. Instruktiv fallen etwa auch die von Auflage zu Auflage vervollkommneten Ausführungen zur Haftungsverfassung ärztlicher Gemeinschaftspraxen und zum Beweiswert einer EDV-Dokumentation aus. Die nicht nur für anwaltliche Berufseinsteiger lesenswerten Muster für eine Klage und Klageerwiderung in Arzthaftungssachen wurden ergänzt. Sämtliche Darstellungen zeichnen sich durch einen eindrucksvollen Tiefgang aus, der das Werk als Handbuch und Nachschlagewerk für Praxis und Wissenschaft gleichermaßen geeignet erscheinen lässt. So wird bei rechtlichen Problemkreisen der Meinungsstand 